Z Herz- Thorax- Gefäßchir 2021 · 35:232-241 https://doi.org/10.1007/s00398-021-00444-z Angenommen: 18. Juni 2021 Online publiziert: 30. Juni 2021

๑) Der/die Autor(en) 2021

\section{Interdisziplinäre Betreuung von Menschen mit Marfan-Syndrom - Pharmakologie, Schwanger- schaft, Auge, Skelett und organisatorische Aspekte}

\author{
Murat Yildiz ${ }^{1}$ Gabor Matyas ${ }^{2} \cdot$ Kerstin Wustmann ${ }^{3}$. Christine Attenhofer Jost ${ }^{4}$. \\ Francesca Bonassin ${ }^{5}$. Beatrice Früh ${ }^{6} \cdot \mathrm{Kan} \mathrm{Min}^{7} \cdot$ Petra Gehle ${ }^{8}$. \\ Giancarlo Bombardieri ${ }^{9} \cdot$ Thierry Carrel $^{1} \cdot$ Florian Schoenhoff' $^{\prime}$ \\ ${ }^{1}$ Klinik für Herz- und Gefäßchirurgie, Universitätsspital Bern, Bern, Schweiz; ${ }^{2}$ Zentrum für \\ Kardiovaskuläre Genetik und Gendiagnostik, Schlieren, Schweiz; ${ }^{3}$ Zentrum für Angeborene Herzfehler, \\ Universitätsspital Bern, Bern, Schweiz; ${ }^{4}$ HerzGefässZentrum Zürich, Klinik im Park, Zürich, Schweiz; \\ ${ }^{5}$ Abteilung für Angeborene Herzerkrankungen, Universitäres Herzzentrum Zürich, Zürich, Schweiz; \\ ${ }^{6}$ Klinik für Augenheilkunde, Universitätsspital Bern, Bern, Schweiz; ' 5 Swiss Scoliosis, Klinik im Park, Zürich \\ Schweiz; ${ }^{8}$ Marfan-Ambulanz, Campus Virchow Klinikum, Charité, Berlin, Deutschland; ${ }^{9}$ Schweizer \\ Marfan-Stiftung, Bern, Schweiz
}

\section{Zusammenfassung}

Im ersten Teil des vorliegenden Beitrags wurde im Detail auf die Genetik, Diagnose, Differenzialdiagnose, die diagnostische Bildgebung, Follow-up, die kardiovaskulären Probleme sowie die Manifestation an Aorta und den großen Gefäßen bei Patienten mit dem Marfan-Syndrom (MFS) eingegangen. In diesem zweiten Teil werden die medikamentöse Therapie des MFS, seine Bedeutung im Zusammenhang mit einer Schwangerschaft, die Beteiligung von Augen und Wirbelsäule bei MFSPatienten sowie die organisatorischen Aspekte einer Marfan-Sprechstunde erläutert. Das MFS ist mit einer Prävalenz von etwa 1-2:10.000 die häufigste hereditäre Bindegewebserkrankung mit vaskulärer Komponente. Ursache sind Mutationen im Gen, das für das extrazelluläre Matrixprotein Fibrillin-1 kodiert. Die Erkrankung wird autosomal-dominant vererbt. Es handelt sich um eine Multisystemerkrankung mit Beteiligung der Aorta, der Mitralklappe, Augen- und Skelettveränderungen. Die Augen- und/oder die Wirbelsäulenbeteiligung sind nicht zu unterschätzen, da sowohl die Skoliose als auch die Linsendislokation etwa $60 \%$ der Patienten mit MFS betreffen. Bis anhin konnte kein Wirkstoff einen klaren Vorteil hinsichtlich klinischer Ereignisse in MFS-Patienten zeigen. Es besteht jedoch der allgemeine Konsens, Patienten mit MFS einen $\beta$-Rezeptoren- oder Angiotensinrezeptorblocker isoliert oder als Kombinationstherapie zu verabreichen. Bei bestehendem Kinderwunsch muss ein besonderes Augenmerk auf die Planung der Schwangerschaft gelegt werden. Im Fall eines Aortenwurzeldurchmessers $>4-4,5 \mathrm{~cm}$ empfiehlt sich, entweder einen prophylaktischen Aortenwurzelersatz zu erwägen oder von einer Schwangerschaft abzuraten. Eine strukturierte Langzeitbetreuung ist für Patienten mit MFS essenziell.

\section{Schlüsselwörter}

Hereditäre Bindegewebserkrankung · Pharmakologie · Schwangerschaft · Ophthalmologie . Organisatorische Aspekte · Skoliose

QR-Code scannen \& Beitrag online lesen 
Das Risiko für Menschen mit einem Marfan-Syndrom (MFS), an den Folgen eines plötzlichen Ereignisses zu versterben, ist dann am höchsten, wenn dem Betroffenen und seinem Umfeld nicht bewusst ist, dass er an einer angeborenen Bindegewebskrankheit leidet. Je nach untersuchter Patientenpopulation stellt sich auch heute noch ein Drittel der Patienten primär mit einem akuten Dissektionsereignis vor [1]. Sind die Patienten erst einmal in ein spezialisiertes Programm eingebunden, ist das Risiko für ein Ereignis gering [2]. Primäre Ziele in der Betreuung von Patienten mit MFS sind daher die korrekte Diagnosestellung und damit die Verhinderung eines plötzlichen Dissektionsereignisses.

\section{Hintergrund}

Obwohl die Probleme, die die MFS-Patienten in ihrem Alltag beschäftigen aus medizinischer Sicht oft gar nicht so maßgeblich sind, bestimmen sie doch zu einem großen Teil ihre Lebensqualität. Chronische Müdigkeit, Schmerzen in den Gelenken, Hernien, Varizen und Probleme in der Schule oder am Arbeitsplatz müssen daher genauso konsequent behandelt werden wie das Aortenwurzelaneurysma.

Das MFS ist von den seltenen Krankheiten eine der häufigsten. Dennoch ist es für den Nichtspezialisten schwierig, genug Erfahrung in der Klinik oder in der Praxis zu sammeln, um diesen oft komplexen Patientenfällen gerecht werden zu können. Aus diesen Erfahrungen heraus

\section{Abkürzungen}

$\begin{array}{ll}\text { AATS } & \text { American Association for Thoracic } \\ & \text { Surgery } \\ \text { ACC } & \text { American College of Cardiology } \\ A C E & \text { Angiotensin-konvertierendes Enzym } \\ A H A & \text { American Heart Association } \\ A R B & \text { Angiotensinrezeptorblocker } \\ A S V & \text { Ambulante spezialfachärztliche } \\ & \text { Versorgung } \\ C T & \text { Computertomographie } \\ E S C & \text { European Society of Cardiology } \\ F D A & \text { Food and Drug Administration } \\ H R & \text { "Hazard ratio“ } \\ I O L & \text { Intraokularlinse } \\ L D S & \text { Loeys-Dietz-Syndrom } \\ M F S & \text { Marfan-Syndrom } \\ M R T & \text { Magnetresonanztomographie } \\ T G F-\beta & \text { "Transforming growth factor } \beta^{\prime \prime} \\ 95 \%-K I & \text { 95\%-Konfidenzintervall }\end{array}$

hat sich das Konzept der „Marfan-Sprechstunden" entwickelt, die heutzutage an den meisten Zentren Patienten mit verschiedensten Bindegewebserkrankungen behandeln. Diese gibt es in verschiedenen Formen, oft sind sie aus dem historischen Kontext des jeweiligen Hauses gewachsen. Allen gemeinsam ist jedoch ein Netzwerk von Spezialisten, die nicht nur ihr eigenes Teilgebiet vor Augen haben, sondern ihre diagnostischen und therapeutischen Schritte im Gesamtkontext der Erkrankung des Patienten sehen.

Im ersten Teil dieses Beitrags [3] wurde im Detail auf die Genetik, Diagnose, Differenzialdiagnose, die diagnostische Bildgebung, Follow-up, die kardiovaskulären Probleme sowie die Manifestation an Aorta und den großen Gefäßen bei Patienten mit MFS eingegangen. In diesem zweiten Teil werden die medikamentöse Therapie des MFS, seine Bedeutung im Zusammenhang mit einer Schwangerschaft, die Beteiligung von Augen und Wirbelsäule bei MFS-Patienten sowie die organisatorischen Aspekte einer Marfan-Sprechstunde erläutert. Im Folgenden werden Spezialisten aus den verschiedensten Teilgebieten in kurzen Abschnitten die essenziellen Aspekte ihrer Arbeit mit MFS-Patienten vorstellen.

\section{Medikamentöse Therapie}

Das primäre Ziel der medikamentösen Behandlung von Patienten mit einem MFS bzw. mit genetisch bedingten Aortopathien ist die Reduktion der Morbidität und Mortalität thorakaler Aortenaneurysmen und-dissektionen. Als Folge einer besseren Einsicht in die physikalischen und molekularen Mechanismen, die zur Entstehung von Aneurysmen führen, sind in den letzten zwei Jahrzehnten spezifische Wirkstoffe als Einzel- oder Kombinationstherapie angewandt worden. Dies ist mit der Hoffnung verbunden, die Dilatationsrate der Aorta und/oder das Risiko für Dissektionen positiv zu beeinflussen und somit indirekt auch den Zeitpunkt einer allfälligen Operation hinauszuschieben. Parallel soll die optimale Behandlung der arteriellen Hypertonie erfolgen; diese stellt zusammen mit der vorliegenden molekulargenetischen Variante den Hauptrisikofaktor für Dissektionen bei Patienten mit vererb- baren Aortopathien dar. Die Einführung einer medikamentösen Behandlung sollte in Betracht gezogen werden, wenn 1.) eine Neigung zur oder eine bestehende Aortendilatation, 2.) eine positive Familienanamnese für Dissektionen und/oder 3.) eine mit Aortendilatation/-dissektion assoziierte Mutation vorliegen/vorliegt. Zur Definition einer relevanten aortalen Dilatation wird bei erwachsenen Patienten ein Z-Score $>2,0$ für Sinusportionen und Aorta ascendens angewandt. Bezüglich der Etablierung der gültigen Normwerte und Z-Scores gelten die Studien von Devereux et al. [4] sowie Campens et al. [5]; für diese sind auch entsprechende interaktive "calculators" zu finden. Auf der Website der Marfan-Foundation (www.marfan.org/dx/ zscore) steht ein Z-Score-Kalkulator für den klinischen Alltag zur Verfügung.

\section{I) Die Therapieinitiierung wird ab Z-Scores $\geq 2,0$ bei Erwachsenen nach Sicherung der Diagnose empfohlen}

Aktuelle Empfehlungen, ab welchem Z-Score eine medikamentöse Therapie bei MFS-Patienten gestartet werden soll, gibt es nicht. Die Autoren empfehlen eine Initiierung ab einem Z-Score $\geq 2,0$ bei erwachsenen Patienten nach Sicherung der Diagnose. Schwieriger ist es bei Kindern und Jugendlichen. Einmal eine medikamentöse Therapie angefangen, gibt es keinen geeigneten Zeitpunkt, diese zu stoppen. In diesen Fällen wird eine medikamentöse Therapie mit der Familie ab einem Z-Score $\geq 2,0$ diskutiert und auf den Beginn einer Therapie $a b$ einem Z-Score $\geq 3,0$ gedrängt.

Gemäß den 2010 erschienenen Guidelines des American College of Cardiology (ACC), der American Heart Association (AHA) und der American Association for Thoracic Surgery (AATS) zu Diagnose und Behandlung von Patienten mit thorakalen Aortenerkrankungen werden heutzutage für Patienten mit MFS und Aortendilatation immer noch $\beta$-Blocker als medikamentöse Erstlinientherapie empfohlen [6]. Diese Empfehlung basiert auf einer kleinen randomisierten „Open-label“-Studie, die eine signifikante Reduktion der Aortenwurzeldilatationsrate und einen Trend zu weniger negativen Ereignissen in der Propra- 
nololbehandlungsgruppe zeigte [7]. Nach dieser ersten Publikation haben sich im Verlauf die lang wirksamen Präparate Atenolol und Metoprolol als Therapiestandard etabliert, wobei in weiteren Studien eine Reduktion klinisch relevanter Ereignisse nicht mehr dokumentiert werden konnte. In Analogie zu den Erwachsenen werden $\beta$-Blocker auch für Kinder mit einer Aortendilatation empfohlen, die Studienlage bietet hierfür allerdings gar keine Evidenz an.

Als medikamentöse Behandlung in der Schwangerschaft sind nur Metoprolol oder Labetalol erlaubt, da Atenolol potenziell negative Auswirkungen auf den Fetus aufweist.

Nach der Publikation der Resultate des Mausmodells für MFS und mit dem Erlangen eines besseren Verständnisses der molekularen Mechanismen, die zu hereditären Aortopathien führen, sind die ersten Studien mit dem Angiotensinrezeptorblocker (ARB) Losartan erschienen. Unter Losartangabe zeigten sich im Mausmodell eine eindeutig geringere Fragmentierung der elastischen Fasern und somit eine stabilere Architektur der Aortenwand, was auf eine Blockade der TGF-Signalkaskade zurückzuführen ist [8]. Die Resultate im Mausmodell waren so eindrücklich, dass relativ rasch eine große randomisierte Studie initiiert werden konnte. Im Pediatric Heart Network Trial wurden daher Kinder mit MFS für Atenolol oder Losartan randomisiert. Die Ergebnisse zeigten jedoch keine eindeutigen Vorteile von Losartan gegenüber Atenolol. Kritiker der Studie bemängelten, dass jeweils eine hohe Dosis Atenolol mit einer eher geringen Dosis Losartan verglichen wurde. Da es keine Placebogruppe gab, können auch keine fundierten Aussagen über die Wirksamkeit der beiden Medikamente insgesamt getroffen werden [9, 10]. In weiteren Studien [11, 12] konnte nur eine Reduktion der Dilatationsrate der Aorta, aber keine positiven Auswirkungen auf Mortalität und klinisch relevante Ereignisse gefunden werden. Als Alternative zu Losartan wird derzeit das Ansprechen auf Irbesartan analysiert. Die definitiven Resultate stehen allerdings noch aus. Es besteht keine Evidenz für die Anwendung weiterer Medikamentenklassen, wie z.B. ACEHemmern, außer als Teil der Behandlung einer vorliegenden arteriellen Hypertonie.
Zuletzt ist erwähnenswert, dass Hinweise dafür vorliegen, dass gewisse Medikamentenklassen, wie z.B. Kalziumantagonisten und Hydralazin, sich potenziell negativ hinsichtlich aortaler Ereignisse auswirken. Auch Antibiotika der Chinolonklasse scheinen aortale Ereignisse zu triggern, weshalb die FDA kürzlich eine Warnung hinsichtlich ihres Gebrauchs bei MFS-Patienten veröffentlichte. Andere bekannte Genmutationen betreffend besteht immer noch keine klare Empfehlung hinsichtlich einer medikamentösen Behandlung, mit Ausnahme des vaskulären Ehlers-DanlosSyndroms, bei dessen Vorliegen sich der $\beta$-Blocker Celiprolol als Therapie der Wahl etabliert hat [13].

Zusammenfassend hat bisher kein Wirkstoff einen klaren Vorteil hinsichtlich klinischer Ereignisse bei MFSPatienten gezeigt. Es besteht ein allgemeiner Konsens, dass eine Therapie mit einem $\beta$-Blocker oder ARB isoliert oder als Kombination sinnvoll ist. Aufgrund des vorteilhaften Nebenwirkungsprofils beginnen viele Zentren bei therapienaiven Patienten mit einem Sartan, insbesondere wenn eine stärkere blutdrucksenkende Wirkung angestrebt wird. Die Vor- und Nachteile sowie die Evidenz einer medikamentösen Therapie sollen individuell abgewogen und mit dem Patienten klar besprochen werden.

\section{Schwangerschaft}

Frauen mit MFS haben ein deutlich erhöhtes Risiko, während der Schwangerschaft eine Aortendissektion oder -ruptur (3\%, Range 1-10\%) zu erleiden, insbesondere wenn der Durchmesser der Aortenwurzel $>4,0 \mathrm{~cm}$ beträgt [14]. Aortendissektionen treten am häufigsten im 3. Trimenon und in der frühen postpartalen Periode auf. Als verantwortlich werden insbesondere die mit einer Schwangerschaft verbundenen hormonellen Veränderungen und die hämodynamische Belastung betrachtet. Die Hauptdeterminante für das Auftreten von Komplikationen ist allerdings das Vorliegen einer relevanten Dilatation der Aorta als Baseline. Bei einem Aortenwurzeldurchmesser $>4,5 \mathrm{~cm}$ empfiehlt sich, entweder einen prophylaktischen Aortenwurzelersatz zu erwägen oder von einer Schwangerschaft abzuraten. Bei einem
Durchmesser zwischen 4,0 und $4,5 \mathrm{~cm}$ sollen das Risiko und das Prozedere individuell, abhängig von anderen Risikofaktoren, wie z. B. positive Familienanamnese für aortale Ereignisse, abgewogen werden [15]. Die amerikanischen Guidelines [6] sind strenger und raten bereits bei einem Durchmesser $>4,0 \mathrm{~cm}$ zum prophylaktischen Aortenwurzelersatz, was diesen Graubereich zwischen 4,0 und $4,5 \mathrm{~cm}$ entstehen lässt.

\section{॥ Bei bestehendem Kinderwunsch sollte eine interdisziplinäre präkonzeptionelle Beratung erfolgen}

Vor der Planung einer Schwangerschaft sollte MFS-Patientinnen eine gründliche interdisziplinäre präkonzeptionelle Beratung in einem tertiären Zentrum mit Expertise angeboten werden. Eine komplette, aktuelle kardiologische Abklärung mit Echokardiographie und Schnittbildgebung (MRT/CT) der gesamten Aorta ist indiziert. Es sollten zudem eine geburtshilfliche Konsultation und idealerweise eine genetische Beratung stattfinden, um die häufigsten geburtshilflichen Komplikationen (v. a. Zervixinsuffizienz/vorzeitige Öffnung des Muttermundes), die Risiken für den Fetus und die Wahrscheinlichkeit einer Vererbung (50\%iges Risiko) ausführlich zu diskutieren [16].

Aus medikamentöser Sicht sollen die Gaben von ARB und ACE-Hemmern mindestens 3 Monate vor dem Eintreten einer Schwangerschaft aufgrund des potenziellen teratogenen Risikos sistiert werden. Dies ist aber lediglich eine Expertenempfehlung bei fehlender Datenlage. Die Gaben von $\beta$-Blockern dürfen und sollten weitergeführt werden. Als medikamentöse Behandlung der ersten Wahl gelten Metoprolol (Maximaldosis idealerweise in 2 bis 3 Einnahmen/Tag aufgeteilt) oder Labetalol. Eine Atenololtherapie wird während der Schwangerschaft ungern weitergeführt, da es potenziell zu einer Beeinträchtigung des fetalen Wachstums kommen kann. Im Fall einer Umstellung der medikamentösen Therapie muss der Blutdruck engmaschig überwacht werden, insbesondere wenn eine bekannte arterielle Hypertonie vorliegt. 


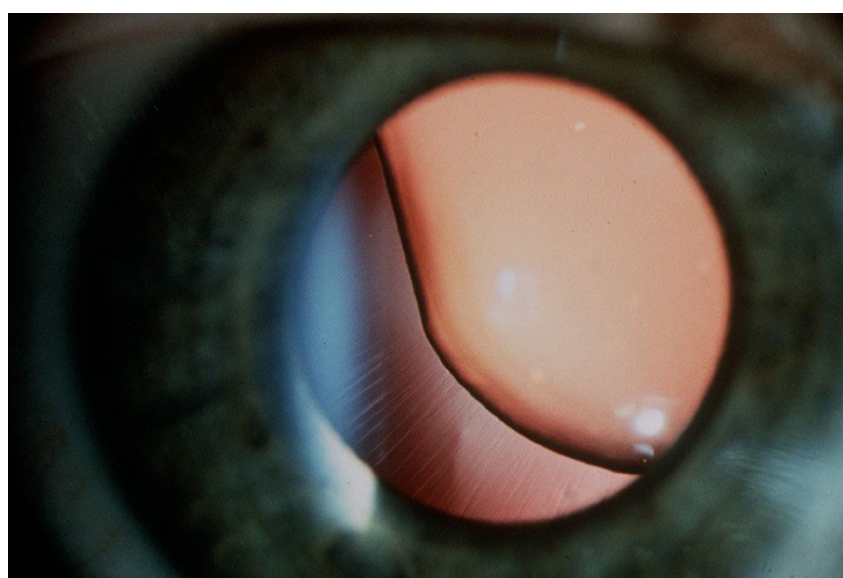

Abb. $1 \triangleleft$ Nach oben und temporal subluxierte klare Linse. Die Linse weist auch ein Kolobom auf; die rarifizierte, elongierte Zonulafaser ist erkennbar

Kardiologische Kontrollen mit echokardiographischer Ausmessung der aortalen Dimensionen sollen während der Schwangerschaft alle 4 bis 8 Wochen erfolgen, insbesondere wenn der Durchmesser der Aortenwurzel $>4,0 \mathrm{~cm}$ beträgt. Elektive MRT-Untersuchungen der Aorta während der Schwangerschaft sollten ohne Gabe von Gadoliniumkontrastmittel durchgeführt werden, da sich dies potenziell teratogen auswirken kann.

Die Geburt soll in einem tertiären Zentrum mit dem Back-up eines interdisziplinären Teams, bestehend aus Kardiologie, kardiovaskulärer Chirurgie, Angiologie und interventioneller Radiologie, geplant werden. Bei einem Aortenwurzeldurchmesser $<4,0 \mathrm{~cm}$ kann eine spontane Geburt angestrebt werden. Dabei empfiehlt sich die frühzeitige Einlage einer Epiduralanästhesie, um den Geburtsstress zu verringern. Bei einem Durchmesser $>4,5 \mathrm{~cm}$ wird eher eine primäre Sectio vorgezogen. Zwischen einem Aortenwurzeldurchmesser $\geq 4,0 \mathrm{~cm}$ und $\leq 4,5 \mathrm{~cm}$ ist das Vorgehen, abhängig vom individuellen Risikoprofil der Patientin, festzulegen [15].

Im Fall einer Typ-A-Dissektion vor der 30. Schwangerschaftswoche sollte eine sofortige Operation durchgeführt werden. Nach der 30. Schwangerschaftswoche wird die Sectio, gefolgt von der Operation an der Aorta, empfohlen [15].

Engmaschige Kontrollen und die Gabe von $\beta$-Blockern sind bis 3 Monate postpartum empfohlen, da eine Dissektion auch noch in dieser Zeit gehäuft vorkommen kann. In der Studie von Roman et al. [17] ereigneten sich 5 von 7 Aortendissektionen in der Post-partum-Periode.
Es ist anzunehmen, dass Frauen während der Schwangerschaft mit vorausgegangener Dissektion ein höheres Risiko für eine Komplikation an der Aorta aufweisen, obwohl die Datenlage diesbezüglich limitiert ist. Aktuell wird Patientinnen mit Z.n. Dissektion meist von einer Schwangerschaft abgeraten. Aus Sicht der Autoren muss dies unter Berücksichtigung des Alters der Dissektion, der Wachstumsrate, der absoluten Größe der Aorta sowie der Dringlichkeit des Kinderwunsches mit der Patientin in einem interdisziplinären Umfeld diskutiert werden, bevor generell von einer Schwangerschaft abgeraten wird.

\section{Augenbeteiligung}

Die häufigste okuläre Manifestation des MFS ist die Linsendislokation (ca. 60\% der Fälle), die durch geschwächte Zonulafasern verursacht ist. Diese kann schon sehr früh im Leben auftreten und ist nicht selten das erste Zeichen des MFS-Krankheitsbildes überhaupt. Kinder mit einer Linsendislokation (meist Subluxation nach oben und temporal, - Abb. 1) haben einen schlechten Visus, sind myop und weisen einen starken mehrheitlich lentikulären Astigmatismus auf. Die Verdachtsdiagnose wird durch aufmerksame Eltern („das Kind nimmt alles sehr nahe zum Schauen“) oder durch Pädiater im Rahmen von Routineuntersuchungen (bei Kleinkindern sind wegen Reflexen keine Messungen mit dem Refraktometer möglich oder schlechter Visus bei älteren Kindern) gestellt. Die opthalmologische Versorgung ausgeprägter Linsensubluxationen ist auch in geübten Händen schwierig: Da der Astigmatismus nicht korneal bedingt ist, nützen Kontaktlinsen wenig. Die volle Astigmatismuskorrektur mithilfe einer Brille ist oft nicht möglich und die Messung der objektiven Refraktion erschwert. Indikationen für die chirurgische Sanierung sind: fehlende Visusverbesserung trotz konsequentem Tragen der Brille und so starke Astigmatismuszunahme, dass die Bestimmung der Stärke unmöglich ist.

Die chirurgische Versorgung ist anspruchsvoll, da die Schwäche der Zonulafaser die Linsenabsaugung erschwert und eine Intraokularlinsen(IOL)-Implantation in den Kapselsack verunmöglicht. Es sind folgende Verfahren möglich:

- das Auge aphak lassen und die Linsenlosigkeit mithilfe von dicken Aphakiegläsern oder Kontaktlinsen korrigieren. Dies hat die Vorteile, dass die Operationsdauer relativ kurz ist und die Langzeitresultate bei Aphakie bekannt sind. Als große Nachteile sind die schlechte postoperative Sehschärfe und v.a. die Abhängigkeit von Kontaktlinsen zu nennen.

- Sulcus-Naht-Implantation einer IOL. Die visuellen Resultate sind exzellent. Nachteil: längere Operationsdauer mit großem Schnitt in der Sklera; theoretisch bestehen die Gefahren der Fadenlyse und nach Jahren einer Luxation der IOL in den Glaskörper.

- Sulcus-Klebung einer IOL. Auch für Erwachsene sind keine Langzeitresultate bekannt.

- Kapselspannring und IOL im Kapselsack. Theoretisch ist dies die beste Methode, da sich die IOL am anatomisch korrekten Ort befindet. Aber dies ist nur bei schwachen Zonuladehiszenzen möglich (meist ist in diesen Fällen noch keine Operationsindikation gegeben). Mit dem Fortschreiten der Zonulaschwäche wird die IOL trotz des Kapselspannrings sicherlich dezentrieren.

Weitere Augenmanifestationen können nebst Linsenschlottern, Myopie und Astigmatismus auch Irisschlottern, Irisatrophie, eine enge Pupille, die schlecht dilatiert, ein Glaukom und eine Netzhautablösung sein. Glaukom und Netzhautablösung sind eher Folgen der Myopie oder von Operationen als primär durch das MFS bedingt. 


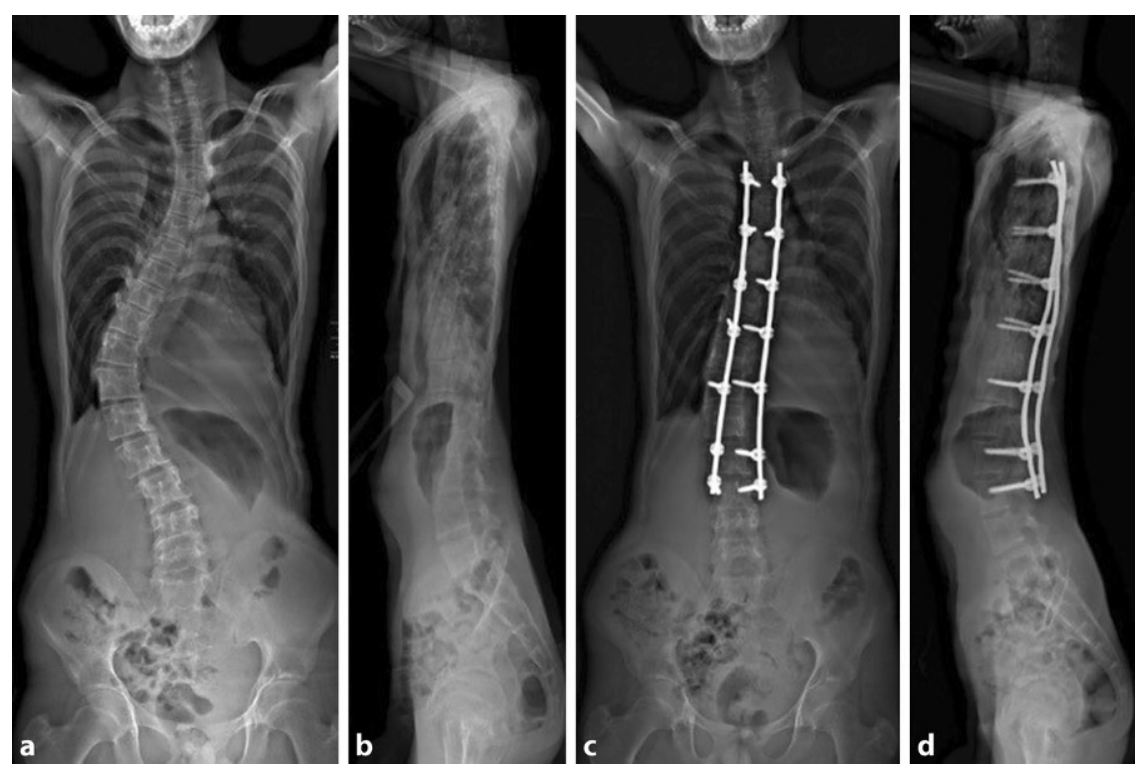

Abb. 2 \ Präoperative Röntgenbilder von einem typischen Marfan-Syndrom-Patienten mit Skoliose (a) und ausgeprägter thorakaler Lordose (b); postoperative Röntgenbilder nach Korrektur von Skoliose (c) und Lordose (d)

Das frühe Erkennen der Linsensubluxation ist sehr wichtig, um eine Amblyopie zu vermeiden. Daher sollte jedes Kind mit einem MFS regelmäßig ophthalmologisch kontrolliert werden. Umgekehrt ist es so, dass jedes Kind mit spontaner Linsensubluxation bis zum Beweis des Gegenteils als MFS-Patient angesehen werden sollte. Beachte: Familiäre Linsendislokationen ohne MFS sind bekannt, aber viel seltener als bei MFS-Patienten.

Die Häufigkeit der ophthalmologischorthoptischen Kontrollen hängt vom Alter des Kindes und der Schwere der Erkrankung ab: Wenn die Linse nicht disloziert ist, genügt anfänglich eine Kontrolle alle 6 Monate und dann einmal im Jahr. Bei Vorliegen einer Dislokation sind die Kontrollen alle 3 bis 6 Monate nötig.

\section{Wirbelsäulenprobleme}

Bei mindestens $60 \%$ der Patienten mit einem MFS liegt eine Skoliose vor - überwiegend thorakal, weniger im thorakolumbalen Bereich ([18, 19]; - Abb. 2). Die Skoliose ist mehrheitlich atypisch, im Vergleich zur verbreiteten Form der idiopathischen Skoliose. Eine Korsettbehandlung ist bei MFSPatienten in der Mehrzahl der Fälle nicht erfolgreich. Die Skoliose ist meistens progressiv. Bei einer Skoliose von mehr als $50^{\circ}$ wird die Indikation zur Korrekturoperation gestellt. Begleitend zur vorliegenden Wirbelsäulendeformitätkann eine Lordose der Brustwirbelsäule bestehen. Eine Lordose in der Brustwirbelsäule sowie auch das nicht selten vorhandene Pectus excavatum verursachen eine Einengung des Brustkorbs, woraus eine Einschränkung der Lungenfunktion resultiert. Um diesezu verbessern, muss die thorakale Kyphose mithilfe einer operativen Skoliosekorrektur rekonstruiert werden.

Eine gründliche, multidisziplinäre Abklärung und präoperative Vorbereitung sind unentbehrlich. Zusätzlich zur kardiopulmonalen Abklärung ist, zur Beurteilung von intraspinalen Missbildungen, eine MRT-Untersuchung der ganzen Wirbelsäule nötig.

Operativ muss die gesamte Krümmung der Wirbelsäule langstreckig von posterior mit Schrauben fixiert und versteift werden. Bei MFS-Patienten sind kurze Versteifungen nicht zu empfehlen - jede skoliotische Kurve der Wirbelsäule sollte instrumentiert werden [20]. Eine selektive Instrumentation der größten Hauptkurve durchzuführen, mit der Erwartung, dass sich die kleineren Nebenkurven spontan bessern, wie bei einer idiopathischen Skoliose üblich, ist bei MFS-Patienten nicht erfolgversprechend. Ein anteriorer Eingriff, entweder zur Diskektomie oder zur Instrumentation, ist bei MFS-Patienten nicht empfoh- len, da dieser mit hohen Risiken assoziiert ist. Erhöhter Blutverlust, Implantatversagen, Pseudarthrose und durale Verletzungen beim Vorliegen einer duralen Ektasie sind bekannte Komplikationen im Rahmen der operativen Skoliosekorrektur bei MFSPatienten.

\section{I) Jede skoliotische Wirbelsäu- lenkurve des MFS-Patienten sollte instrumentiert werden}

Im Fall einer Operationsindikation bei sehr jungen Kindern ( $<10$ Jahren) kann eine Versteifung herausgezögert werden, in dem vorerst ein verlängerbares Implantat (",growing rod") eingesetzt wird. Dies erlaubt das weitere Wachstum des instrumentierten Bereichs der Wirbelsäule, bis die Kinder für die definitive Versteifung alt genug sind. Das Growing rod kann entweder mechanisch oder magnetisch sein. Mechanische Growing rods müssen wiederholt (im Durchschnitt einmal/Jahr) im Rahmen einer kurzen Anästhesie operativ verlängert werden, die magnetischen dagegen lassen sich in der Sprechstunde ohne Anästhesie mithilfe eines magnetischen Geräts von außen verlängern.

Die durale Ektasie, eine Erweiterung des Nervenkanals durch pathologische Ausweitung des Duralsacks, liegt bei $60-90 \%$ der MFS-Patienten vor ([21, 22];

- Abb. 3). Diese ist größtenteils im lumbalen Bereich lokalisiert und verursacht eine Ausdünnung der Pedikel sowie Laminae. Manchmal entstehen anteriore Meningozelen hinter dem Peritoneum sowie große Duralzysten in den Foramina. Die duralen Ektasien sind üblicherweise asymptomatisch und selten mit lageabhängigen Kopfschmerzen verbunden. Bei ausgeprägten Ektasien können bis zu 50\% der Patienten unter lumbalen Rückenschmerzen leiden. Für die Instrumentation der Wirbelsäule stellen diese anatomischen Veränderungen Probleme dar. Die dünnen Pedikel im lumbalen Bereich erschweren oder verunmöglichen sogar das Einbringen von Pedikelschrauben. Zudem sind die dünnen Laminae so schwach, dass eine alternative Fixationsmöglichkeit mithilfe von Laminahaken auch nicht möglich ist. In solchen Fällen muss die Instrumentation der Wirbelsäule bis in das Becken vorgenommen werden, da eine stabile 

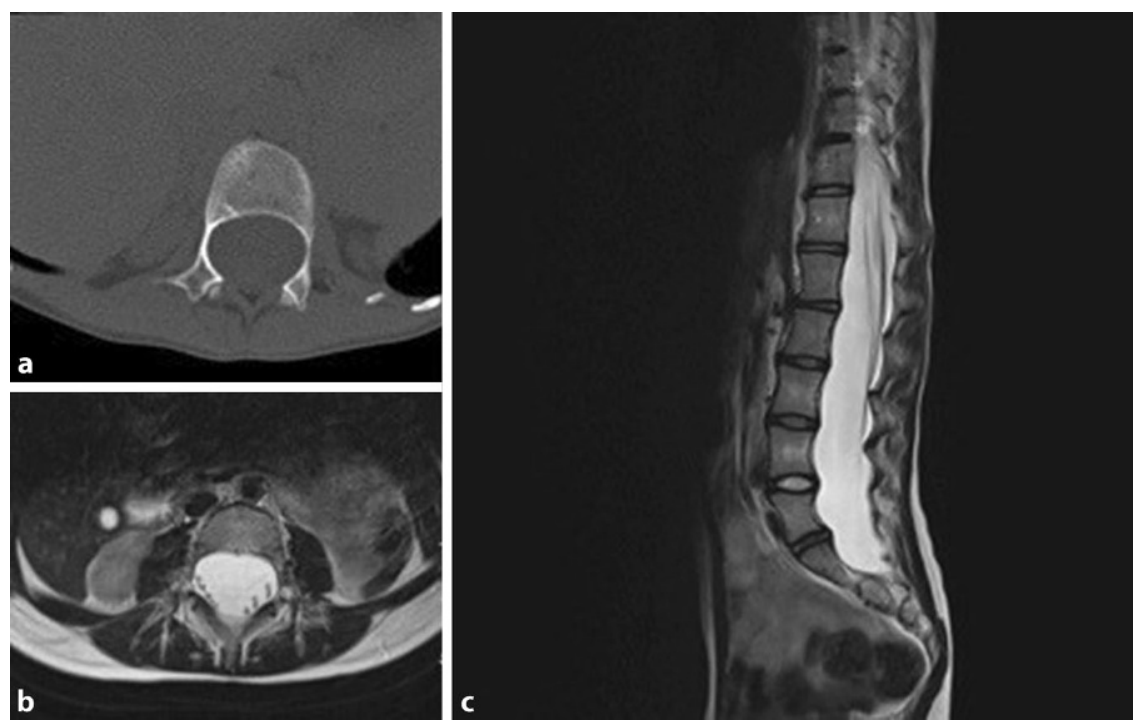

Abb. $3 \Delta$ Ausgeprägte Ektasie der Dura mater im lumbalen Bereich bei einem Marfan-Syndrom-Patienten. $\mathbf{a}, \mathbf{b}$ In Horizontalebene, $\boldsymbol{c}$ in Sagitalebene

Verankerung in der Lendenwirbelsäule nicht erreichbar ist. Bei $6 \%$ der Patienten mit MFS treten Spondylolisthesen auf. Dabei handelt es sich am meisten um „High-grade"-Spondylolisthesen, auch kombiniert mit dysplastischen Veränderungen des lumbosakralen Übergangs und Wirbelkanalstenose. Bei Kindern mit MFS ist die Spondylolisthese progressiv, weshalb wegen der Schmerzen und der Progression in den meisten Fällen eine Operation notwendig ist. Der Eingriff besteht in der Spinalkanaldekompression, Reposition der Spondylolisthese und einer Spondylodese im lumbosakralen Übergang.

Es weisen $16 \%$ der MFS-Patienten eine Kyphose in der Halswirbelsäule auf. Kinder haben häufig eine ligamentäre Instabilität im okzipitozervikalen Übergang, was bei einer Operationsvorbereitung berücksichtigt werden muss [23]. Aufgrund dessen sollen die Kinder mit MFS auf Sportarten, die die Halswirbelsäule stark belasten, verzichten.

\section{Aufbau einer Marfan- Sprechstunde}

Die ambulante spezialfachärztliche Versorgung (ASV) hat 2014 die seit 2009 bestehende Regelung der spezialfachärztlichen Sprechstunde in Krankenhäusern nach $\S 116 \mathrm{~b}$ des Fünften Buches Sozialgesetzbuch (SGBV) abgelöst. An der ASV kön- nen auch niedergelassenen Fachärzte, medizinische Versorgungszentren und Teams von ermächtigten Ärzten in Krankenhäusern teilnehmen, sofern sie die jeweiligen Anforderungen erfüllen ( $\bullet$ Tab. 1). Diese sind in der ASV-Richtlinie (zuletzt aktualisiert am 17.12.2020, in Kraft getreten am 06.05.2021) und der jeweiligen Anlage definiert.

Ziel ist es, dass Patienten mit einigen wenigen seltenen Krankheiten oder schweren Krankheitsverläufen, die in der Richtlinie konkretisiert wurden, durch eine enge Verzahnung von Spezialisten verschiedener Fachdisziplinen besser versorgt werden. Die Behandlung übernimmt ein Team, dem je nach Indikation ganz bestimmte Fachärzte und auch Psychotherapeuten angehören. Dieses Team besteht aus der Teamleitung, einem Kernteam sowie weiteren Fachärzten, die bei Bedarf hinzugezogen werden.

\section{I) Ziel ist die bestmögliche Patientenversorgung durch eine enge Verzahnung verschiedener Experten}

Die Teamleitung übernimmt die fachliche und organisatorische Koordination. Die Mitglieder des Kernteams sind Fachärzte, die aufgrund ihrer Kenntnisse und Erfahrungen bei der Behandlung der jeweiligen Erkrankung mitwirken. Die dritte Ebene bilden die hinzuzuziehenden Fachärz- te, deren Kenntnisse bei einem Teil der Patienten ergänzend benötigt werden.

Der erweiterte Landesausschuss der kassenärztlichen Vereinigungen prüft die Zugangsvoraussetzungen, die für die Diagnosegruppe der genetischen Aortenkrankheiten auch beinhalten, dass die ASV-Ambulanz mindestens 50 Patienten im Jahr behandelt. Zum Erreichen der optimalen hochspezialisierten Behandlung gilt die Pflicht zur höchstpersönlichen Leistungserbringung durch den jeweiligen im Team mitarbeitenden Facharzt. Abgerechnet wird nach dem Einheitlichen Bewertungsmaßstab (EBM) der niedergelassenen Ärzte.

Patienten mit genetischen Aortenkrankheiten, zu denen auch das MFS zählt, benötigen regelmäßig Zugang zu aufwendigen Untersuchungen und Therapien. Daher kann der Besuch in der ASVAmbulanz eine deutliche Verbesserung der Betreuung und eine fachübergreifende ganzheitliche Therapie in der bisherigen medizinischen Versorgung etablieren. Dieses Ziel kann v.a. dann erreicht werden, wenn alle Komponenten der ASV gut ineinandergreifen.

Als zentrale Voraussetzung für eine professionelle und erfolgreiche medizinische Versorgung in der ASV zählen neben den verschiedenen Fachdisziplinen, die sich inhaltlich/fachlich und kommunikativ eng miteinander abstimmen müssen, auch gut durchdachte Verwaltungsabläufe, die die fachärztlichen Leistungserbringer so weit wie möglich entlasten. Eine eng verzahnte Kommunikation zwischen der ASV und den Hausärzten ist wechselseitig erforderlich. Die erfordert eine ausführliche schriftliche Befundmitteilung. Aufgrund der Anforderung der höchstpersönlichen Leistungserbringung durch die beteiligten Fachärzte ist eine Zuarbeit durch Assistenzärzte ausgeschlossen. Hierdurch entfällt beispielsweise eine rotierende Ausbildung der Assistenzärzte in Spezialwissen über die genetischen Aortenkrankheiten, die sich gerade in den ASV-Ambulanzen mit der hohen Konzentration der Patienten mit seltenen Krankheiten anbieten würde. Einschränkend sind noch weitere Limitationen und Nachteile zu nennen:

An erster Stelle steht für viele Patienten diegrößere Entfernung zum spezialisierten Zentrum, häufig verbunden mit der Not- 
Tab. 1 Voraussetzungen für Vertragsärzte und Krankenhäuser gemäß der Richtlinie des Gemeinsamen Bundesausschusses über die ambulante spezialfachärztliche Versorgung (ASV) nach §116b SGBV, Anlage Marfan-Syndrom,

iK 2021-05-06.pd

Personelle Anforderungen
Tätigkeit in einem interdisziplinären Behandlungsteam (Zusammensetzung s. rechte Spalte). Erfüllung von Qualitätsanforderungen von Qualitätsvereinbarungen nach $\S 135$ Abs. 2 SGB V, sofern diese für Leistungen des Behandlungsumfangs zutreffen

Mindestmengen: Das Kernteam muss mindestens 50 Patienten der oben genannten Diagnosen (Verdacht oder gesichert) pro Jahr in der laufenden ASV behandeln, wobei im ersten Jahr der ASV-Teilnahme eine Unterschreitung bis zu $50 \%$ möglich ist. Im Jahr vor der ASVAnzeige müssen die Zahlen zu 50\% nachgewiesen werden
Interdisziplinäres Team

Ein Team der folgenden Zusammensetzung ist Voraussetzung für die ASV-Berechtigung im Bereich Marfan-Syndrom

a) Teamleitung

Die Teamleitung kann entweder der Facharzt für Herzchirurgie, für innere Medizin, der Kardiologe bzw. der Kinderkardiologe übernehmen

\section{b) Kernteam}

- Herzchirurgie

- Innere Medizin und Kardiologie oder

Sofern Kinder und Jugendliche behandelt werden sollen: Kinder- und Jugendmedizin mit Schwerpunkt Kinderkardiologie

- Orthopädie und Unfallchirurgie

c) Hinzuzuziehende Fachärzte

- Augenheilkunde

- Gynäkologie

- Gefäßchirurgie

- Humangenetik

- Innere Medizin und Pneumologie (bei der Behandlung von Kindern und Jugendlichen kann alternativ ein Kinderarzt mit Schwerpunkt Kinderpneumologie benannt werden)

- Laboratoriumsmedizin

- Radiologie

- Psychiatrie und Psychotherapie oder psychosomatische Medizin und Psychotherapie oder psychologische oder ärztliche Psychotherapeutin oder psychologischer oder ärztlicher Psychotherapeut (bei Behandlung von Kindern und Jugendlichen alternativ auch ein Facharzt für Kinderpsychiatrie und -psychotherapie)

Prozessuale Vor-

Zusammenarbeit mit sozialen Diensten sowie Physiotherapeuten

aussetzungen wendigkeit, sich in einem großen Krankenhaus zurechtzufinden. Die Ärzte sind nicht bekannt und haben keinen Kontakt zum Hausarzt. Durch Konzentration auf wenige Zentren entstehen lange Wartezeiten, auf den Termin und auf die jeweiligen Untersuchungen an den Behandlungsterminen. In den ASV-Ambulanzen kommt es aufgrund der niedrigen Zugangsschwelle zu Kapazitätsgrenzen, die aber bei seltenen Krankheiten zur Erhöhung der Diagnosewahrscheinlichkeit erforderlich ist. Die Abrechnung erfolgt wie in der Praxis beim niedergelassenen Hausarzt nach dem EBMKatalog, der die notwendige umfangreiche persönliche Gesprächszeit mit dem spezialisierten Facharzt der ASV nicht annähernd abbildet. In der interdisziplinären Sprechstunde werden die einzelnen möglichen abrechenbaren Leistungen, bezogen auf das jeweilige Fachgebiet, durch die exklusive Festlegung erschwert.

Die Ausschließlichkeit der vorgegebenen Leistungen, die in der Anlage für jede konkretisierte Diagnosegruppe zur ASV facharztgebunden festgeschrieben ist, bil- det zwar eine gute Grundlage für einen „Routinefall", jedoch sind Ausnahmeregelungen für die tatsächlich im individuellen Fall notwendigen und sich in der Praxis entwickelnden optimalen Diagnose- und Therapiestrategien erforderlich. Hier gestaltet sich jedoch die Struktur der Zulassung und Zulassungsprüfung über den Gemeinsamen Bundesausschuss und die jeweiligen erweiterten Landesausschüsse als ein sehr starres Gebilde.

\section{Sicht der Patienten und Patientenvereinigungen in der Schweiz}

Die Marfan Stiftung Schweiz, die mittlerweile seit über 30 Jahren aktiv ist, ist stets bemüht, die Betroffenen zu beraten und zu unterstützen. Diese Aufgabe wird mithilfe persönlicher Telefon- und Internetkommunikation sowie durch formelle Informationsveranstaltungen wahrgenommen. Die Informationsveranstaltungen sind vorwiegend medizinisch orientiert und werden von den jeweiligen Spezialisten bestritten.
Des Weiteren werden informelle Treffen angeboten, in denen sich die Betroffenen und Angehörige gegenseitig austauschen können. Im Fall von betroffenen Kindern besteht das Bestreben, die Lehrerschaft und Mitschüler über die Krankheit zu informieren. Zur Bekanntmachung des Syndroms wird auch mit Medienmitteilungen operiert.

Die Herausforderung bei der Beratung ist es sicherzustellen, dass die Betroffenen den Zugang zu einer medizinischen Betreuung haben, d.h., primär von einem fachkompetenten Hausarzt begleitet werden. Darüber hinaus wird sich auf den Support der Marfan-Sprechstunden gestützt, die quasi als "oberste Instanz" in der Schweiz für die MFS-Patienten zur Verfügung steht und eine interdisziplinäre Betreuung bietet. Zudem besteht erfreulicherweise inzwischen ein landesweites Spezialistennetzwerk, das untereinander koordiniert und sich bezüglich schwieriger Fälle austauscht. 


\section{Fazit für die Praxis}

- Zur medikamentösen Therapie des MFS ist die Gabe eines $\beta$-Rezeptoren- oder Angiotensinrezeptorblockers (ARB) isoliert oder als Kombination sinnvoll. Vor- und Nachteile einer medikamentösen Therapie sollen individuell abgewogen und mit dem Patienten klar besprochen werden.

- Frauen mit MFS haben ein deutlich erhöhtes Risiko, während einer Schwangerschaft eine Aortendissektion oder -ruptur zu erleiden. Die Hauptdeterminante für das Auftreten von Komplikationen ist eine bereits vorliegende relevante Aortendilatation. Bei einem Aortenwurzeldurchmesser $>4,5 \mathrm{~cm}$ empfiehlt es sich, einen prophylaktischen Aortenwurzelersatz zu erwägen oder von einer Schwangerschaft abzuraten. Bei einem Durchmesser zwischen 4,0 und $4,5 \mathrm{~cm}$ sollen das Risiko und das Prozedere individuell, abhängig von anderen vorliegenden Risikofaktoren, beurteilt werden.

- Das frühe Erkennen der häufigsten okulären Manifestation, der Linsensubluxation, ist wichtig, um eine Amblyopie zu vermeiden. Jedes Kind mit einem MFS sollte regelmäßig ophthalmologisch kontrolliert werden, und jedes Kind mit spontaner Linsensubluxation sollte bis zum Beweis des Gegenteils als MFS-Patient angesehen werden.

- Mindestens $60 \%$ der MFS-Patienten weisen eine überwiegend thorakale Skoliose, die meistens progressiv verläuft, auf. Bei einer Ausprägung von mehr als $50^{\circ}$ ist die Indikation zur Korrekturoperation gegeben. Begleitend können eine Lordose der Brustwirbelsäule und ein Pectus excavatum auftreten. Diese verursachen eine Einengung des Brustkorbs mit konsekutiver Einschränkung der Lungenfunktion. In diesen Fällen muss die thorakale Kyphose operativ korrigiert werden.

\section{Korrespondenzadresse}

\section{PD Dr. Florian Schoenhoff}

Klinik für Herz- und Gefäßchirurgie, Universitätsspital Bern

Freiburgstr. 18, 3010 Bern, Schweiz

florian.schoenhoff@insel.ch

Funding. Open access funding provided by University of Bern

\section{Einhaltung ethischer Richtlinien}

Interessenkonflikt. M. Yildiz, G. Matyas, K. Wustmann, C. Attenhofer Jost, F. Bonassin, B. Früh, K. Min P. Gehle, G. Bombardieri, T. Carrel und F. Schoenhoff geben an, dass kein Interessenkonflikt besteht.
Für diesen Beitrag wurden von den Autoren keine Studien an Menschen oder Tieren durchgeführt. Für die aufgeführten Studien gelten die jeweils dort angegebenen ethischen Richtlinien.

Open Access. Dieser Artikel wird unter der Creative Commons Namensnennung 4.0 International Lizenz veröffentlicht, welche die Nutzung, Vervielfältigung, Bearbeitung, Verbreitung und Wiedergabe in jeglichem Medium und Format erlaubt, sofern Sie den/die ursprünglichen Autor(en) und die Quelle ordnungsgemäß nennen, einen Link zur Creative Commons Lizenz beifügen und angeben, ob Änderungen vorgenommen wurden.

Die in diesem Artikel enthaltenen Bilder und sonstiges Drittmaterial unterliegen ebenfalls der genannten Creative Commons Lizenz, sofern sich aus der Abbildungslegende nichts anderes ergibt. Sofern das betreffende Material nicht unter der genannten Creative Commons Lizenz steht und die betreffende Handlung nicht nach gesetzlichen Vorschriften erlaubt ist, ist für die oben aufgeführten Weiterverwendungen des $\mathrm{Ma}$ terials die Einwilligung des jeweiligen Rechteinhabers einzuholen.

Weitere Details zur Lizenz entnehmen Sie bitte der Lizenzinformation auf http://creativecommons.org/ licenses/by/4.0/deed.de.

\section{Literatur}

\section{Verwendete Literatur}

1. Schoenhoff FS, Jungi S, Czerny M, Roost E, Reineke D, Matyas G, Steinmann B, Schmidli J, Kadner A Carrel T (2013) Acute Aortic Dissection Determines the Fate of Initially Untreated Aortic Segments in Marfan Syndrome. Circulation 127:1569-1575 https://doi.org/10.1161/CIRCULATIONAHA.113. 001457

2. Guillaume J, Delphine D, Florence T, Florence $A$ Olivier M, Francois R, Gabriel D, Lea M, Laura K, Dalil H, Christophe B, Carine R, Alec V, Catherine B (2012) Aortic event rate in the Marfan population. Circulation 125:226-232. https://doi.org/10.1161/ CIRCULATIONAHA.111.054676

3. Yildiz M, Matyas G, Wustmann K, Attenhofer Jost $C$, Bonassin F, Früh B, Min K, Gehle P, Bombardieri G, Carrel T, Schönhoff F (2020) Interdisziplinäre Betreuung von Menschen mit Marfan-Syndrom - Genetik, bildgebende Untersuchung und kardiovaskuläres System. Z Herz Thorax Gefasschir 34:115-126. https://doi.org/10.1007/s00398020-00364-4

4. Devereux RB, De Simone G, Arnett DK, Best LG, Boerwinkle E, Howard BV, Kitzman D, Lee ET, Mosley TH, Weder A, Roman MJ (2012) Normal limits in relation to age, body size and gender of two-dimensional echocardiographic aortic root dimensions in persons >15 years of age. Am J Cardiol 110:1189-1194. https://doi.org/10.1016/j. amjcard.2012.05.063

5. Campens L, Demulier L, De Groote K, Vandekerckhove K, De Wolf D, Roman MJ, Devereux RB, De Paepe A, De Backer J (2014) Reference values for echocardiographic assessment of the diameter of the aortic root and ascending aorta spanning all age categories. Am J Cardiol 114:914-920

6. Hiratzka LF, Bakris GL, Beckman JA, Bersin RM, Carr VF, Casey DE, Eagle KA, Hermann LK, Isselbacher EM, Kazerooni EA (2010) 2010
ACCF/AHA/AATS/ACR/ASA/SCA/SCAI/SIR/STS/SVM guidelines for the diagnosis and management of patients with thoracic aortic disease. J Am Coll Cardiol 55:e27-e129

7. Shores J, Berger KR, Murphy EA, Pyeritz RE (1994) Progression of aortic dilatation and the benefit of long-term $\beta$-adrenergic blockade in Marfan's syndrome. NEngl J Med 330:1335-1341

8. Habashi JP, Judge DP, Holm TM, Cohn RD, Loeys BL, Cooper TK, Myers L, Klein EC, Liu G, Calvi C, Podowski M, Neptune ER, Halushka MK, Bedja D, Gabrielson K, Rifkin DB, Carta L, RamirezF, Huso DL, Dietz HC (2006) Losartan, an AT1 antagonist, prevents aortic aneurysm in a mouse model of Marfan syndrome. Science 312:117-121. https:// doi.org/10.1126/science.1124287

9. Lacro RV, Dietz HC, Sleeper LA, Yetman AT, Bradley TJ, Colan SD, Pearson GD, Selamet Tierney ES, Levine JC, Atz AM (2014) Atenolol versus losartan in children and young adults with Marfan's syndrome. N Engl J Med 371:2061-2071

10. Erbel R, Aboyans V, Boileau C, Bossone E, Bartolomeo RDi, Eggebrecht H, Evangelista A, Falk V, Frank H, Gaemperli O, Grabenwöger M, Haverich A, lung B, Manolis AJ, Meijboom F, Nienaber CA, Roffi M, Rousseau H, Sechtem U, Sirnes PA, Allmen RS von, Vrints CJM, (CPG) ESCC for PG, Zamorano $\mathrm{JL}$, Achenbach S, Baumgartner $\mathrm{H}, \mathrm{Bax} J \mathrm{~J}$, Bueno $H$, Dean V, Deaton C, Erol Ç, Fagard R, Ferrari R, Hasdai D, Hoes A, Kirchhof P, Knuuti J, Kolh P, Lancellotti P, Linhart A, Nihoyannopoulos P, Piepoli MF, Ponikowski P, Sirnes PA, Tamargo JL, Tendera $M$, Torbicki A, Wijns W, Windecker $S$, reviewers D, Nihoyannopoulos P, Tendera M, Czerny M, Deanfield J, Mario C Di, Pepi M, Taboada MJS, Sambeek MR van, Vlachopoulos C, Zamorano JL, Grimm M, Musayev O, Pasquet A, Kušljugić Z, Cikes M, Georghiou GP, Stasek J, Molgaard H, Kõvask; S, Kytö V, Jondeau G, Bakhutashvili Z, von Kodolitsch Y, Tsioufis C, Temesvári A, Rubinshtein R, AntoniniCanterin F, Lunegova O, Stradins P, Chammas E, Jonkaitiene R, Cassar A, Bjørnstad K, Widenka K, Sousa Uva M, Lighezan D, Perunicic J, Madaric J, Vilacosta I, Bäck M, Mahdhaoui A, Demirbag R, Kravchenko I (2014) 2014 ESC Guidelines on the diagnosis and treatment of aortic diseases: Document covering acute and chronic aortic diseases of the thoracic and abdominal aorta of the adult. The Task Force for the Diagnosis and Treatment of Aortic Diseases of the European So. Eur Heart J 35:2873-2926. https://doi.org/10. 1093/eurheartj/ehu281

11. Chiu HH, Wu MH, Wang JK, Lu CW, Chiu SN, Chen CA, Lin MT, Hu FC (2013) Losartan added to $\beta$-blockade therapy for aortic root dilation in marfan syndrome: a randomized, open-label pilot study. Mayo Clin Proc. https://doi.org/10.1016/j. mayocp.2012.11.005

12. Groenink M, den Hartog AW, Franken R, Radonic T, de Waard V, Timmermans J, Scholte AJ, van den Berg MP, Spijkerboer AM, Marquering HA (2013) Losartan reduces aortic dilatation rate in adults with Marfan syndrome: a randomized controlled trial. Eur Heart J 34:3491-3500

13. Ong K-T, Perdu J, De Backer J, Bozec E, Collignon $\mathrm{P}$, Emmerich J, Fauret A-L, Fiessinger J-N, Germain DP, Georgesco G (2010) Effect of celiprolol on prevention of cardiovascular events in vascular Ehlers-Danlos syndrome: a prospective randomised, open, blinded-endpoints trial. Lancet 376:1476-1484

14. Smith K, Gros B (2017) Pregnancy-related acute aortic dissection in Marfan syndrome: a review of the literature. Congenit Heart Dis 12:251-260 
15. Regitz-Zagrosek V, Roos-Hesselink JW, Bauersachs J, Blomström-Lundqvist $C$, Cífková R, De Bonis $M$, lung $B$, Johnson MR, Kintscher U, Kranke P, Lang IM, Morais J, Pieper PG, Presbitero P, Price S, Rosano GMC, Seeland U, Simoncini T, Swan L, Warnes CA (2018) 2018 ESC Guidelines for the management of cardiovascular diseases during pregnancy. Eur Heart J 39:3165-3241. https://doi. org/10.1093/eurheartj/ehy340

16. Meijboom LJ, Drenthen W, Pieper PG, Groenink M, van der Post JAM, Timmermans J, Voors AA, Roos-Hesselink JW, van Veldhuisen DJ, Mulder BJM (2006) Obstetric complications in Marfan syndrome. Int J Cardiol 110:53-59

17. Roman MJ, Pugh NL, Hendershot TP, Devereux RB, Dietz H, Holmes K, Eagle KA, LeMaire SA, Milewicz DM, Morris SA (2016) Aortic complications associated with pregnancy in Marfan syndrome: the NHLBI national registry of genetically triggered thoracic aortic aneurysms and cardiovascular conditions (gen TAC). J Am Heart Assoc 5:e4052

18. Sponseller PD, Hobbs W, Pyeritz RE (1995) The thoracolumbar spine in Marfan syndrome. J Bone Joint Surg Am 77:867-876

19. Jones KB, Erkula G, Sponseller PD, Dormans JP (2002) Spine deformity correction in Marfan syndrome. Spine 27:2003-2012

20. Gjolaj JP, Sponseller PD, Shah SA, Newton PO, Flynn JM, Neubauer PR, Marks MC, Bastrom TP (2012) Spinal deformity correction in Marfan syndrome versus adolescent idiopathic scoliosis: learning from the differences. Spine 37(18):1558-1565

21. PyeritzRE, FishmanEK, Bernhardt BA, Siegelman SS (1988) Dural ectasia is a common feature of the Marfan syndrome. Am J Hum Genet 43:726

22. Mesfin A, Ahn NU, Carrino JA, Sponseller PD (2013) Ten-year clinical and imaging follow-up of dural ectasia in adults with Marfan syndrome. Spine J 13:62-67

23. Hobbs WR, Sponseller PD, Weiss A-PC, Pyeritz RE (1976) The cervical spine in Marfan syndrome. Spine 22:983-989

\section{Weiterführende Literatur}

24. Loeys BL, Dietz HC, Braverman AC, Callewaert BL, De Backer J, Devereux RB, Hilhorst-Hofstee $Y$, Jondeau G, Faivre L, Milewicz DM (2010) The revised Ghent nosology for the Marfan syndrome. JMed Genet 47:476-485

25. Mongeon F-P, Marcotte F, Terrone DG (2016) Multimodality noninvasive imaging of thoracic aortic aneurysms: time to standardize? Can J Cardiol 32:48-59. https://doi.org/10.1016/j.cjca.2015.09.025

26. Judge DP, Dietz HC (2005) Seminar: Marfan's syndrome. Lancet 366(9501):1965-1976. https:// doi.org/10.1016/S0140-6736(05)67789-6
Interdisciplinary care of people with Marfan syndrome-Pharmacology, pregnancy, eye, skeleton and organizational aspects

In the first part of this article the genetic basis, diagnosis, differential diagnosis, diagnostic imaging, follow-up, cardiovascular problems and manifestations in the aorta and the great vessels in patients with Marfan syndrome were discussed in detail. In this second part the pharmacological treatment, pregnancy-related issues, eye involvement and involvement of the spine as well as the organizational aspects of a Marfan consultation in patients with Marfan syndrome are explained. Marfan syndrome is the most frequent hereditary disease of connective tissue with vascular components and has a prevalence of 1-2:10,000. Marfan syndrome is caused by mutations in the gene encoding the extracellular matrix protein fibrillin-1. It is inherited in an autosomal dominant fashion. It is a multisystemic disease with involvement of the aorta and mitral valve with ocular and skeletal alterations. The involvement of the eye and/or the spine should not be underestimated as approximately $60 \%$ of patients with Marfan syndrome are affected by scoliosis as well as lens dislocation. Up until now no substance could show a clear advantage with respect to the clinical results in patients with Marfan syndrome; however, there is a general consensus that treatment with a beta blocker or angiotensin receptor blocker alone or as a combination treatment should be initiated in patients with Marfan syndrome. Particular attention should be paid when planning a pregnancy. If the aortic root reaches a diameter $>4-4.5 \mathrm{~cm}$, it is recommended that a prophylactic replacement of the aortic root should be considered or patients should be advised against pregnancy. Providing a structured long-term follow-up is essential for patients with Marfan syndrome.

\section{Keywords}

Hereditary disease of connective tissue - Pharmacology · Pregnancy · Ophthalmology · Organizational aspects · Scoliosis 
Hier steht eine Anzeige.

黑 Springer 